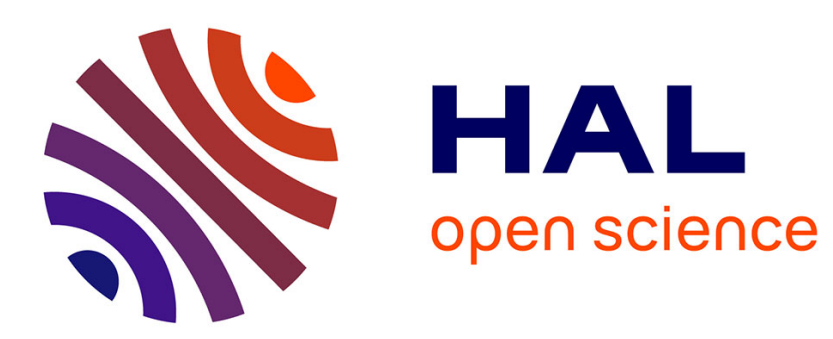

\title{
Performances of Density Functional Tight-Binding Methods for Describing Ground and Excited State Geometries of Organic Molecules
}

Arnaud Fihey, Denis Jacquemin

\section{- To cite this version:}

Arnaud Fihey, Denis Jacquemin. Performances of Density Functional Tight-Binding Methods for Describing Ground and Excited State Geometries of Organic Molecules. Journal of Chemical Theory and Computation, 2019, 15 (11), pp.6267-6276. 10.1021/acs.jctc.9b00688 . hal-02364861

HAL Id: hal-02364861

https://hal-univ-rennes1.archives-ouvertes.fr/hal-02364861

Submitted on 3 Feb 2020

HAL is a multi-disciplinary open access archive for the deposit and dissemination of scientific research documents, whether they are published or not. The documents may come from teaching and research institutions in France or abroad, or from public or private research centers.
L'archive ouverte pluridisciplinaire HAL, est destinée au dépôt et à la diffusion de documents scientifiques de niveau recherche, publiés ou non, émanant des établissements d'enseignement et de recherche français ou étrangers, des laboratoires publics ou privés. 


\title{
Performances of Density Functional
}

\section{Tight-Binding Methods for Describing Ground and Excited State Geometries of Organic}

\section{Molecules}

\author{
Arnaud Fihey ${ }^{*, \dagger}$ and Denis Jacquemin $*, \ddagger$ \\ †Institut des Sciences Chimiques de Rennes, UMR 6226 CNRS, Université de Rennes 1, 263 \\ Av. du Général Leclerc, 35042, Cedex Rennes, France \\ $\ddagger$ Laboratoire CEISAM - UMR CNRS 6230, Université de Nantes, 2 Rue de la Houssinière, \\ BP 92208, 44322 Nantes Cedex 3, France \\ E-mail: Arnaud.Fihey@univ-rennes1.fr; Denis.Jacquemin@univ-nantes.fr \\ Phone: +32 (0) 2232373 58; +32 (0) 251125564
}

\begin{abstract}
We present a benchmark of the performances of Density Functional Tight-Binding model (DFTB) and its Time-Dependent counterpart (TD-DFTB) in describing both the ground state (GS) and excited state (ES) geometries of a panel of 30 organic molecules. Thanks to high-level wavefunction reference calculations, we are able to quantitatively assess the strengths and weaknesses of four DFTB models, using either a second- or third-order Self-Consistent Charges procedures, as well as different sets of parameters. The performances of the different DFTB models are found to be largely dependent on the type of bond considered, but the global mean absolute error remains acceptable for such "cheap" quantum mechanic calculations, as it slightly exceeds the
\end{abstract}


one obtained with DFT (PBE and B3LYP) or CC2 models for the GS of the same set of compounds. When considering the ES of the molecules, the TD-DFTB errors are surprisingly not systematically larger than their GS counterparts. However, the trends, either when going from the GS to the ES, or within a homologous chemical series, are less consistently reproduced with DFTB than with the $a b$ initio models. This work therefore validates the use of TD-DFTB for describing ES geometries while highlighting that care has to be applied when looking at subtle variations.

\section{Introduction}

Modeling photo-induced phenomena in large organic compounds over long timescales remains a key challenge in computational chemistry. Indeed, this requires the description of experimental systems encompassing photoactive molecule(s) within a complex environment, i.e., interacting with a grafting support (surface, nanoparticle), an embedding matrix (solvent, polymers), or other molecules in their vicinity. The fields of photovoltaics, sensing for medical applications, and optical information storage stand as typical examples in which the excited state (ES) events should be understood and controlled. If the vertical ES properties of isolated organic molecules (encompassing up to ca. 100 atoms) can nowadays be described with TimeDependent Density Functional Theory (TD-DFT) for a reasonable accuracy/effort ratio, 12 exploring the excited state potential energy surfaces of large compounds rapidly becomes out of reach for $a b$ initio approaches. In this context, the use of the so-called "DensityFunctional Tight-Binding" (DFTB) framework ${ }^{3 / 4}$ appears to be an appealing alternative. DFTB is a quantum mechanics semi-empirical scheme, that is rooted in DFT and inherits its versatility, while allowing saving ca. $99 \%$ of the computational effort by pre-computing (at the DFT/PBE level of theory) and tabulating, as parameters, the two-electrons integrals needed to solve the Kohn-Sham equations. DFTB can be viewed as an improvement of the well-known Hückel model, as matrix elements are already known, making the calculation task reduced to a matrix diagonalization and eigenvalues search problem. Among the different 
versions of DFTB available, the most popular formalism is the Self-Consistent Charges DFTB (SCC-DFTB) .516$]$ It relies on the variation of the atomic charges to iteratively search for the answer of the tight-binding problem while allowing charge transfer to occur between atoms as well as between orbitals of the same atom. This self-consistent modification of the DFTB energy with atomic charges is determined through a second or third order Taylor expansion of the DFT energy around a reference density (usually the sum of atomic densities), leading respectively to the SCC-DFTB2 $2^{\frac{5}{5}}$ and SCC-DFTB $3^{\frac{78}{78}}$ models, simply denoted "DFTB2" and "DFTB3" in the following.

The accuracy of DFTB methods is by construction depending on the quality of the parameterization used and a specific application should ideally require its own set of parameters. Nevertheless, one can expect the SCC-DFTB parameters to be transferable to a certain extent. Within this duality, both specific and general parameter sets have been proposed during the last two decades and one can now study inorganic compounds,, 910 organic systems, 11112 as well as hybrid organic-inorganic materials. $\frac{13114}{1}$ Concerning organic molecules, the most

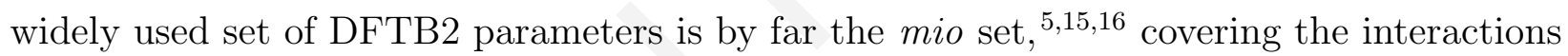
between C, H, O, N, S, and P. Ground state (GS) properties have been extensively tested with the DFTB2/mio approach: the obtained geometries and electronic structure properties are generally in good to very good agreement with DFT results. ${ }^{11}$ This mio set has been extended to the description of halogen atoms thanks to the development of the halorg parameters.17 Another popular set of parameters is the matsci set that is designed not only to describe inorganic solids with DFTB2 but also hybrid materials encompassing organic molecules. 18119 In this latter set, the interactions between $\mathrm{C}, \mathrm{N}, \mathrm{O}$, and $\mathrm{H}$ are available. More recently, the $30 b$ set has been developed to work together with the DFTB3 model and includes parameters for organic atoms $(\mathrm{C}, \mathrm{N}, \mathrm{O}, \mathrm{H}, \mathrm{P}$, and $\mathrm{S}) \stackrel{820}{,}$ alkaline and alkaline earth atoms $(\mathrm{Ca}, \mathrm{K}, \mathrm{Na}$, and $\mathrm{Mg}$ ), as well as halogens (F, Cl, Br, and I) $\stackrel{2122}{21}$ improving the overall accuracy as compared to the DFTB2 sets. Finally, the latest extension of DFTB is the development of formulations relying on range-separated DFT functionals rather than semi-local functionals during the 
parameterization process,,$\sqrt{2324}$ in order to avoid the overdelocalization and spurious electronic states typically observed with DFTB2 in large systems. This new "Long-range Corrected DFTB2" (LC-DFTB2) is today available for a few atoms, namely C, H, N, and O, within the ob2 parameter set designed for organic and biological systems. $\frac{25}{25}$

During the last few years, the SCC-DFTB formalism has been constantly extended, and now includes many of the popular features of DFT including a Time-Dependent version, 26 empirical corrections for dispersion, $\frac{27 / 28}{2}$ and continuum approaches to estimate bulk solvent effects. $\stackrel{29130}{ }$ More specifically, the Time-Dependent DFTB (TD-DFTB) ${ }^{26}$ formalism, that has been available for almost two decades, allows not only bypassing the computational bottlenecks of TD-DFT in the calculation of vertical excitations but also grants more efficient ES geometry optimizations, thanks to the implementation of TD-DFTB analytical gradients. 31 We underline that TD-DFTB calculations do not require any additional parameters besides those needed for the GS, so that the above-cited sets designed for organic molecules can be straightforwardly used to explore various experimental photochemistry problems. However, to date, only a few works have already applied the TD-DFTB model to organic-based compounds, e.g., to compute the absorption spectra of flavonol derivatives interacting with

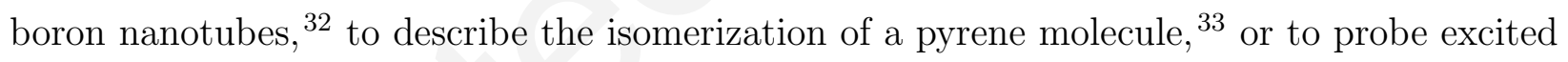
state intramolecular proton transfer ${ }^{34}$ One explanation for this relatively small number of TD-DFTB works is that it constitutes a relatively unexplored computational tool for the community with a yet unclear applicability. Indeed, extensive studies of the TD-DFTB performances remain scarce in the literature, besides the original validations to be found in the theoretical works introducing the TD-DFTB extensions for DFTB2, DFTB3 and LC-DFTB2. 266 30]34 This is especially the case for excited state geometries, for which few

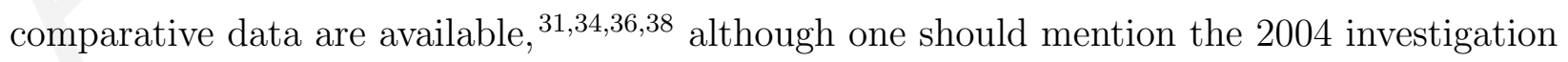
of Wanko et al., who investigated the potential energy surfaces of several hallmark organic molecules (benzene, polyenes, protonated Schiff bases...) with TD-DFTB2. $\stackrel{38}{ }$ They concluded that such level of theory is reliable for the geometries when standard DFT can be trusted, 
and that the two approaches have similar limitations in both accuracy and applicability.

In the present work, we provide an assessment of several DFTB variants for GS and ES geometrical parameters. As in all benchmarks, the quality of the reference values is of prime importance to obtain reliable conclusions. If one can easily find a plethora of experimental GS geometries, this does not hold for the ES, for which the reported "measured" values are often deduced from quite complex fitting procedures of the vibronic spectra, rendering the final error bars non-negligible. For instance, for formaldehyde, a small and intensively investigated compound, the experimentally reported estimates for the puckering angle in the lowest singlet ES are $20.5^{\circ}, \underline{39} 31.1^{\circ}, \stackrel{39}{ }$ and $34.0^{\circ} .40$ Strikingly, the two former values have been obtained in the same work, $\frac{139}{b y}$ analyzing two different bands, $0^{0}$ and $4^{1}$, respectively. In fact, accurate experimental geometries are mostly available for di- and tri-atomics only. Whilst these data have been used as reference, ${ }^{36}$ such compounds remain far from the one used in DFTB applications and, more importantly, these data are too limited to allow determining statistically-relevant conclusions for different types of bonds. Therefore, at least for the ES, it is safer to rely on theoretically-determined geometries. The natural choice would be to select TD-DFT geometries to assess DFTB, an approach indeed applied previously in the original papers introducing the various TD-DFTB models. ${ }^{30131}$ However, in a recent work, one of us has shown that the accuracy of the geometries obtained with TD-DFT (ES) tends to be significantly smaller than with DFT (GS). ${ }^{41}$ We have therefore to select reference geometries determined with highly-accurate wavefunction approaches and large atomic basis sets. It was shown that both the GS and ES structural parameters obtained with CCSDR $(3),{ }^{42}$ CC3, $\stackrel{43144}{ }$ and CASPT2, $\stackrel{45146}{ }$ are highly consistent when selecting active spaces encompassing all valence electrons for the latter approach. ${ }^{47}$ For instance, for the puckering angle in formaldehyde the CCSDR(3), CC3, CASPT2, and CCSDT values are $36.6^{0}, 36.8^{0}, 38.2^{0}$, and $37.3^{0}$, respectively, ${ }^{4147}$ that is, they span over a significantly tighter range of values than their experimental counterparts (vide supra). We therefore select such highly accurate references here. The DFTB GS and ES geometries under study result from 
four different models: DFTB2 associated with the mio set and the matsci set, DFTB3 model using the $3 o b$ set and LC-DFTB2 with the ob2 parameters. The results presented here are first discussed in details for a few representative cases before providing a statistical analysis.

\section{Computational Details}

\subsection{Reference Geometries}

A list of reference geometries obtained at high level of theory for 30 small and medium organic compounds (34 excited states) are gathered in the Supporting Information (SI). We consider here valence singlet ES in closed-shell compounds, a selection justified as DFTB is designed for such states in larger compounds, and not for Rydberg or open-shell states. The vast majority of our reference geometrical parameters are extracted from Refs. 41, 47, and 48. These parameters are mainly determined at the CC3 or CCSDR(3) levels of theory using large basis sets, CASPT2 reference structures being used when significant multi-reference effects are at play. Large triple- $\zeta$ basis sets, namely, def2-TZVPP and aug-cc-pVTZ have been used in all cases. In Tables S1-S7 in the SI, we report a few additional/improved reference values obtained here. The methods, algorithms, and programs used to obtain these new data are exactly the same as in our previous works. $\frac{41 \mid 47 / 48}{4}$ We therefore refer the interested readers to these previous investigations for further technical details.

\subsection{DFTB calculations}

All DFTB2/DFTB3 and TD-DFTB2/TD-DFTB3 calculations are conducted using the 18.2 version of the DFTB+ software, ${ }^{49}$ while the LC-DFTB2 and LC-TD-DFTB2 results were obtained using a developement version of GAMESS-US containing the new implementation of the range-corrected formalism recently published by Nishimoto. 30

For both the GS and the ES, all geometry optimizations are made using the reference wavefunction geometries as starting point and frequency calculations are performed to assess 


\subsection{Ab initio Calculations}

To provide a fair assessment of the quality of DFTB with respect to other methods, we have also compared our reference geometrical parameters to TD-(PBE) ${ }^{[52}$ (TD-)B3LYP $53 \sqrt[56]{56}$ and 
(LR-)CC2 $2^{\frac{43157 / 58}{5}}$ results for all compounds. Please note that PBE is the functional used to parametrize DFTB2 and DFTB3 and is thus included here as a reference for a more direct evaluation of potential pitfalls of the tight-binding approach originating from the underlying DFTs. Full results are available in the SI. Some PBE, B3LYP and CC2 values could be taken from literature (see the SI), but new calculations have to be made in the present work. All our DFT computations are performed with the Gaussian16 code with the aug-cc-pVTZ basis set. ${ }^{[5]}$ We first optimize both the GS and ES geometries (starting with the coordinates obtained at the reference level of theory) until the residual mean square force is below the 1 $\times 10^{-5}$ au threshold, before analytically determining the vibrational frequencies to ascertain the nature of the minima (when an instability is noted, this is indicated in the SI). These calculations use a $(99,590)$ pruned integration grid (ultrafine) and rely on an improved energy convergence threshold (ca. $10^{-9}$ au). The CC2 calculations are done with Turbomole, 60 selecting the aug-cc-pVTZ basis set and taking advantage of the resolution-of-identity (RI) approach. During the CC2 calculations, the SCF, second-order, and geometry optimization thresholds are respectively tightened to $10^{-9} \mathrm{au}, 10^{-7}$ au and $10^{-5}$ au, and all electrons are correlated. Numerical vibrational frequency calculations are also performed at the CC2 level of theory.

\section{Results and Discussion}

\subsection{Selected examples}

Before discussing in the next Section the statistical results obtained with DFTB2/mio, DFTB2/matsci, DFTB3/3ob, and LC-DFTB2/ob2, and comparing them to DFT and CC2 data, let us briefly present a few representative examples. First in the vast majority of cases, it is possible to obtain both the GS and ES structures with all DFTB approaches without specific difficulties. There are a few exceptions to this general statement. For both formylchloride and phosgene, the optimizations of the lowest ES lead to an unphysical extension of the 
C-Cl bond(s) with DFTB, which is incorrect as these ES have been characterized in gas phase experimentally. ${ }^{6163}$ It is noteworthy that the same dissociative behavior is obtained with B3LYP for formylchloride (but no phosgene), whereas coupled cluster approaches (and TD-DFT with many other functionals) are more successful. 1147 In some cases, the symmetry of the ES differs in the reference approach and in DFTB. An interesting case is nitrosomethane, a molecule for which experimental evidences indicate an eclipsed GS conformation but a

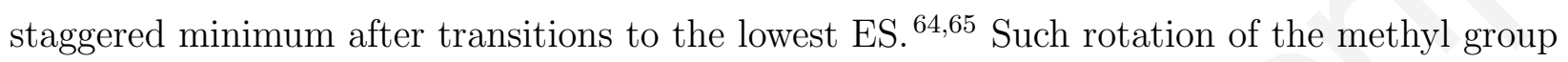
upon excitation is accurately reproduced by PBE, B3LYP, CC2, and CC3 (Table S32 in the SI). The same holds for (LC-)DFTB2 (with all parameter sets), but not with DFTB3/3ob that yields an imaginary frequency for the eclipsed GS, an error that can be corrected by using a H-X (X: heavy atom) damped interaction approach (see computational details). In this case the presence of the oxygen atom relatively close to one of the hydrogen atoms of the methyl may hint at potential weak interactions missing or incompletely described in DFTB3 without additional damping parameter. However, the fact that DFTB2 is able to retrieve the correct conformations renders quite unclear the need of higher level electrostatic perturbation models, and pinpoints instead to some error compensation phenomenon. For thioformylchloride, the reference experimental work ${ }^{66}$ as well as high-level $\mathrm{CC}^{47}$ and multi-reference ${ }^{\sqrt{4767}}$ calculations point out at a slight puckering of the ES, which could be qualitatively reproduced with DFTB3/3ob but not with DFTB2/mio that predicts a planar ES (Table S40 in the SI). At the TD-DFT level, the same "flattening" problem is obtained with functionals including a large ratio of exact exchange, e.g., M06-2X and $\omega$ B97X, but not with B3LYP.41

In Table 1 we list selected geometrical parameters for the homologous formaldehyde, acetaldehyde, and acetone series (see SI for complete data). All tested DFTB models correctly foresee a strong elongation of the carbonyl bond lengths accompanied by a significant puckering after the $n \rightarrow \pi^{\star}$ transition. In addition, in the GS, the CO bond length is accurately estimated by all methods, and one notes that LC-DFTB2 provides longer carbonyl lengths than the other DFTB methods, especially for formaldehyde. All approaches also predict 
a slight lengthening of this bond when going from formaldehyde to acetone but this effect seems exaggerated with DFTB $(+0.005 \AA$ according to $\operatorname{CCSDR}(3)$ versus $+0.025 \AA$ with DFTB2/mio). For formaldehyde, the elongation of the bond in the ES is estimated to be $+0.113 \AA$ with $\operatorname{CCSDR}(3)$, a value inline with the most recent experiments, $\stackrel{40 \mid 68}{ }$ as well as with previously reported MR-AQCC $\frac{69}{69}$ and CR-EOM-CCSD $(\mathrm{T})^{\underline{67}}$ studies. Consistently with our previous work, ${ }^{41}$ both PBE and B3LYP slightly underestimates this change, whereas DFTB overestimates it, quite strongly in the case of the DFTB2/matsci parameters $(+0.167$ $\AA$ ). The evolution of this ES lengthening with the addition of methyl groups seems also exaggerated with DFTB as compared to CCSDR(3). For the puckering angle, a parameter that is very sensitive to the level of theory and challenging for experiment (see Introduction), our theoretical best estimates are ca. $37-38^{\circ}$ for formaldehyde and acetaldehyde but $42^{\circ}$ for acetone. Such trend is correctly restored by B3LYP though this functional systematically provides too small angles $\left(33^{-}-34^{\circ}\right.$ and $\left.37^{\circ}\right)$. PBE does not provide such an accurate trend, but delivers errors smaller than $10^{\circ}$ in all cases. All tested DFTB parameterizations provide an incorrect trend with a strong decrease of this puckering upon addition of methyl groups (ca. $-10^{\circ}$ per methyl group). As a consequence, DFTB reasonably estimates the puckering angle in acetaldehyde but returns rather large errors for formaldehyde (overestimation) and acetone (underestimation), with absolute errors larger than with PBE. For acetone, the LC-DFTB2 puckering angle is too small by $25^{\circ}$. This is a first illustration that while DFTB provides, on average, rather accurate geometrical parameters, the evolution in a homologous chemical series, i.e., the consistency, is less satisfying than with standard hybrid DFT approaches.

In Table 2, we present the results obtained for two compounds in which the lowest transition presents a $\pi \rightarrow \pi^{\star}$ character. In benzene, the $\mathrm{CC}$ bond length as well as its elongation upon excitation are consistently given by all approaches, although LC-DFTB2/ob2 yields a slightly too long GS bond length, but an accurate extension in the ES. For the $\mathrm{CH}$ bonds, DFTB accurately predicts negligible changes from the GS to the ES but yields too long GS distances, especially with the two DFTB2 parameterizations, e.g., the GS CH bond 
Table 1: Selected geometrical parameters for formaldehyde, acetaldehyde, and acetone determined with several methods. We provide the $\mathrm{C}=\mathrm{O}$ bond length in the GS, and its elongation in the lowest singlet ES in parenthesis (in $\AA$ ), as well as the ES puckering angle $(\eta$ in degrees).

\begin{tabular}{|c|c|c|c|c|c|c|}
\hline \multirow[b]{2}{*}{ Method } & \multicolumn{3}{|c|}{$\mathrm{C}=\mathrm{O}$} & \multicolumn{3}{|c|}{$\eta$} \\
\hline & $\mathrm{H}_{2} \mathrm{C}=\mathrm{O}$ & $\mathrm{MeHC}=\mathrm{O}$ & $\mathrm{Me}_{2} \mathrm{C}=\mathrm{O}$ & $\mathrm{H}_{2} \mathrm{C}=\mathrm{O}$ & $\mathrm{MeHC}=\mathrm{O}$ & $\mathrm{Me}_{2} \mathrm{C}=\mathrm{O}$ \\
\hline DFTB2/mio & $1.183(+0.137)$ & $1.196(+0.151)$ & $1.208(+0.167)$ & 53.3 & 42.9 & 31.2 \\
\hline DFTB2/matsci & $1.192(+0.167)$ & $1.204(+0.176)$ & $1.216(+0.188)$ & 55.2 & 43.6 & 26.0 \\
\hline DFTB3/3ob & $1.183(+0.121)$ & $1.204(+0.129)$ & $1.215(+0.142)$ & 55.3 & 44.7 & 32.2 \\
\hline LC-DFTB2/ob2 & $1.206(+0.115)$ & $1.211(+0.141)$ & $1.219(+0.135)$ & 40.0 & 33.8 & 17.6 \\
\hline $\mathrm{PBE}$ & $1.210(+0.096)$ & $1.215(+0.103)$ & $1.221(+0.106)$ & 38.6 & 34.0 & 34.5 \\
\hline B3LYP & $1.200(+0.092)$ & $1.204(+0.096)$ & $1.210(+0.096)$ & 33.8 & 33.5 & 36.7 \\
\hline $\operatorname{CCSDR}(3)^{a}$ & $1.207(+0.113)$ & $1.209(+0.118)$ & $1.212(+0.120)$ & 36.6 & 37.9 & 42.3 \\
\hline $\operatorname{Exp}^{b}$ & $1.207(+0.116)$ & $1.21(+0.11)$ & & 34 & 26 & \\
\hline
\end{tabular}

${ }^{a}$ CCSDR(3)/ aug-cc-pVTZ values from Refs. 47, 41, and the present work for formaldehyde, acetaldehyde and acetone, respectively. ${ }^{b}$ Most recent (collection of) experimental data we are aware of: Ref. 40 for formaldehyde and Ref. 70 for acetaldehyde.

length is $1.098 \AA$ with DFTB2/mio but $1.081 \AA$ with CCSDR(3)/def2-TZVPP. For the GS geometry of cyanogen $(\mathrm{N} \equiv \mathrm{C}-\mathrm{C} \equiv \mathrm{N}), \mathrm{DFTB}, \mathrm{PBE}$ and $\mathrm{B} 3 \mathrm{LYP}$ all predict $\mathrm{C} \equiv \mathrm{N}$ bonds with errors of ca. $0.010 \AA$, an acceptable deviation, LC-DFTB2 being significantly more efficient. In contrast, the central CC bond is too long by ca. 0.015-0.025 $\AA$ with DFTB whereas both PBE and B3LYP are close to the spot. As a consequence, the bond length alternation (BLA), an important parameter for evaluating the electronic communication in $\pi$-delocalized systems reaches $0.249 \AA$ with DFTB3/3ob but $0.219 \AA$ with CC3. As for benzene, the evolution of both bond lengths upon electronic transition are quite accurately reproduced with DFTB but for a slight overestimation of the elongation of the $\mathrm{CN}$ triple bonds. Due to a small error compensation, the DFTB BLA are accurate for the ES, with a value of $0.071 \AA$ with DFTB3/3ob close to the CC3 reference value of $0.062 \AA$. This shows that there is not always a degradation of DFTB's accuracy when going the GS to the ES. One also notices for cyanogen, that the DFTB geometrical parameters are not necessarily closer to the ones obtained with PBE (the functional used to parametrize DFTB2 and DFTB3), than to the B3LYP values. In short, DFTB globally shows a satisfying performance for both benzene and cyanogen. 
Table 2: Selected geometrical parameters for benzene and cyanogen determined with several methods. We provide the bond lengths in the GS, and their elongation in the selected ES (see the SI) in parenthesis (in $\AA$ ). TBE is the theoretical best estimate used as reference values.

\begin{tabular}{|c|c|c|c|c|}
\hline \multirow[b]{2}{*}{ Method } & \multicolumn{2}{|c|}{ Benzene } & \multicolumn{2}{|c|}{ Cyanogen } \\
\hline & $\mathrm{CC}$ & $\mathrm{CH}$ & $\mathrm{CN}$ & $\mathrm{CC}$ \\
\hline DFTB2/mio & $1.396(+0.032)$ & $1.098(0.000)$ & $1.149(+0.092)$ & $1.406(-0.081)$ \\
\hline DFTB2/matsci & $1.393(+0.034)$ & $1.102(-0.001)$ & $1.155(+0.098)$ & $1.404(-0.081)$ \\
\hline DFTB3/3ob & $1.396(+0.035)$ & $1.088(0.000)$ & $1.154(+0.098)$ & $1.403(-0.086)$ \\
\hline LC-DFTB2/ob2 & $1.402(+0.031)$ & $1.089(0.000)$ & $1.159(+0.085)$ & $1.395(-0.084)$ \\
\hline PBE & $1.388(+0.031)$ & $1.084(0.005)$ & $1.167(+0.075)$ & $1.374(-0.066)$ \\
\hline B3LYP & $1.391(+0.030)$ & $1.082(-0.002)$ & $1.152(+0.078)$ & $1.376(-0.079)$ \\
\hline $\mathrm{TBE}^{a}$ & $1.396(+0.026)$ & $1.081(-0.002)$ & $1.161(+0.076)$ & $1.380(-0.081)$ \\
\hline
\end{tabular}

${ }^{a}$ CCSDR(3)/def2-TZVPP values of this work for benzene, CC3/aug-cc-pVTZ data from Ref. 47 for cyanogen.

\subsection{Statistical study}

The results of a statistical investigation carried out for all approaches using the CC3 or CCSDR(3) geometries as reference are displayed in Table 3 that reports the mean signed (MSE) and mean absolute (MAE) errors. Note that for two ES ( $B_{u}$ state of acetylene and $A$ " of diazomethane) the CASPT2 geometry is taken as benchmark as significant multi-reference characters appear for these ES. $\frac{47}{}$ A histogram representation of the cumulative errors per bond type is available in Figure 1 .

Let us start our analysis by looking at the PBE, B3LYP and CC2 statistics, for which the present results are globally inline with previous benchmarks focused on TD-DFT or post-HF methods. $\frac{41 / 47 / 71}{75}$ Indeed, it can be seen that: i) a higher accuracy is almost systematically obtained for the GS parameters than for the ES ones; ii) the polar CO, CN, and CX bonds are the one generating the largest errors with these three methods; iii) errors are also notably larger for $\mathrm{CH}$ bonds with $\mathrm{PBE}$; iv) the ES carbonyl bond lengths tend to be significantly too short with B3LYP and PBE but significantly too long with CC2, which is consistent with other investigations; $\frac{47 / 73 / 76 / 77}{\mathrm{v}}$ ) the MAE obtained with the three methods are alike (see Figure 1), so that using CC2 as reference to assess the accuracy of B3LYP (or other similar functionals) is probably a relatively risky approach. The standard deviations of the errors 


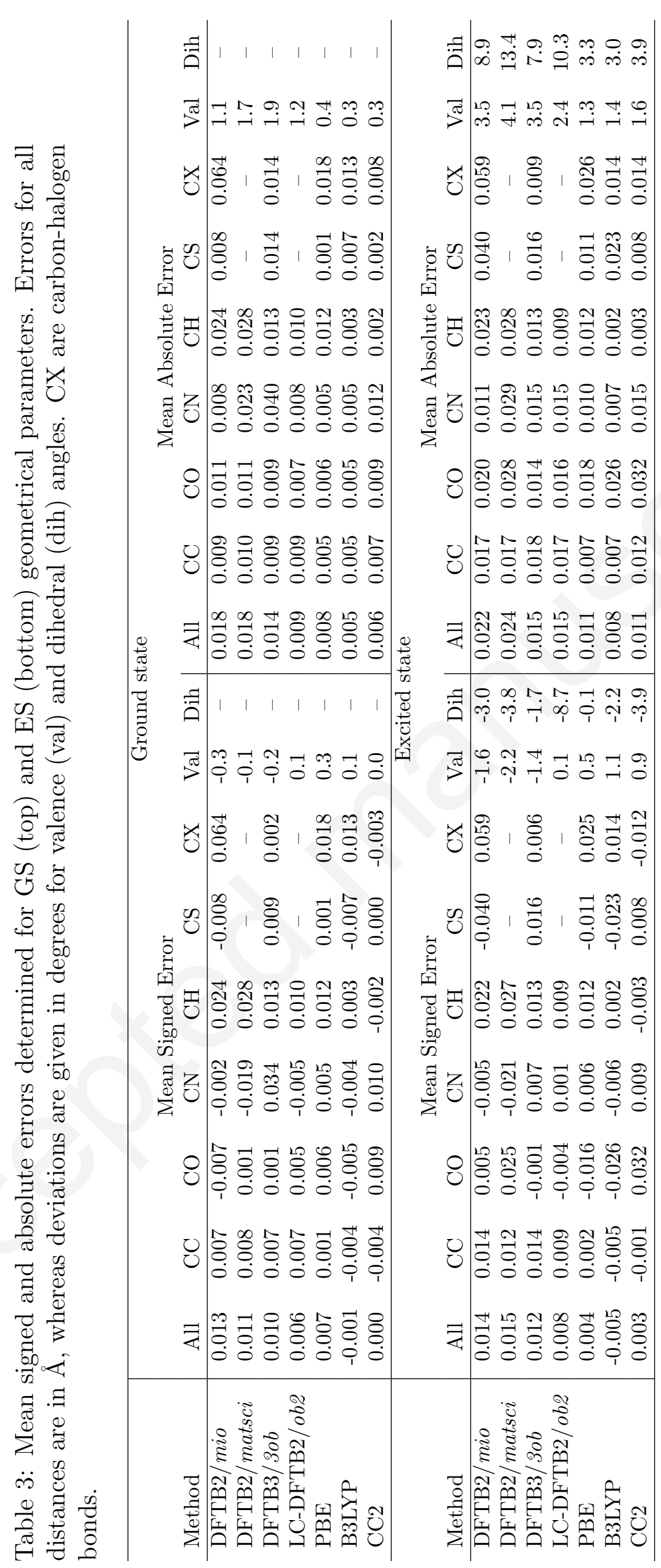


(STD) obtained with PBE, B3LYP and CC2 by considering all bond lengths are respectively 0.007, 0.007 and $0.009 \AA$ in the GS, and $0.015,0.013$ and $0.019 \AA$ in the ES. These small STD indicate rather consistent estimates, especially in the GS.
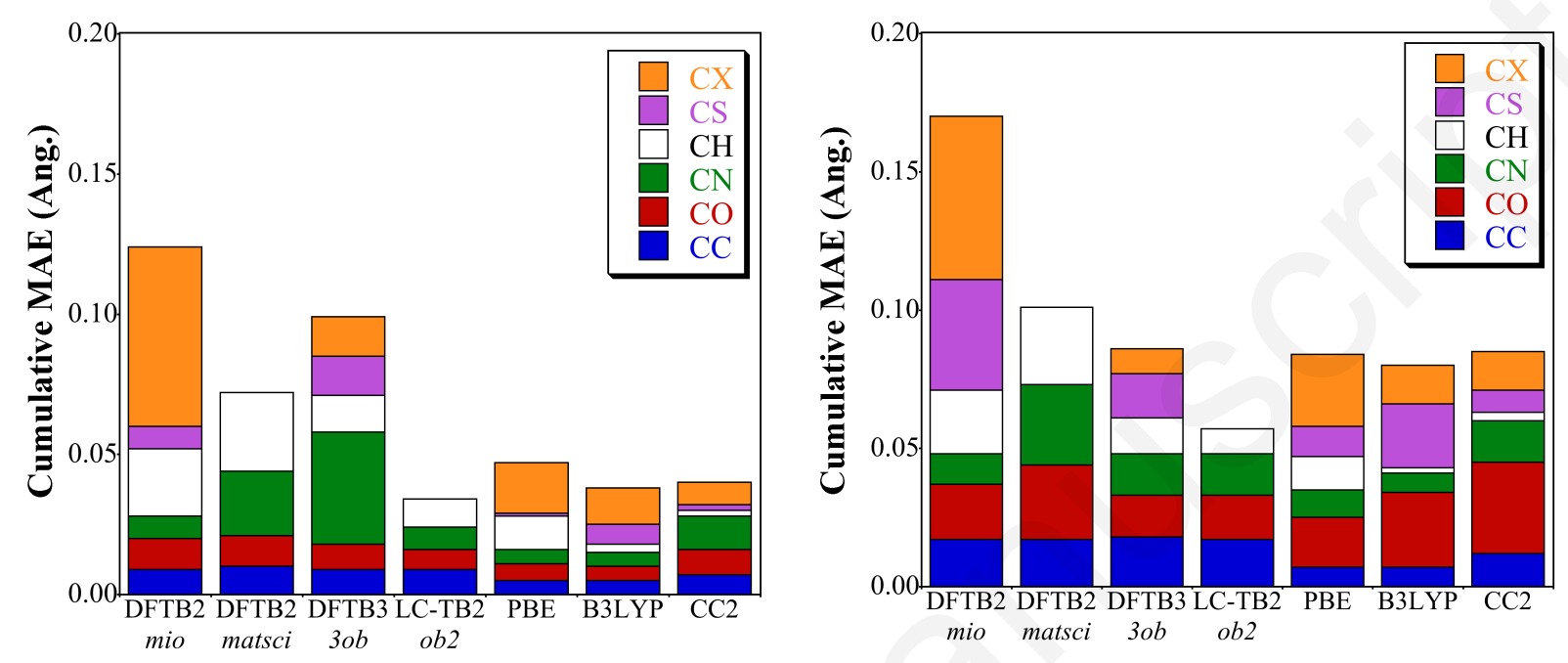

Figure 1: Cumulative mean absolute errors for the GS (left) and ES (right) for six kinds of bond lengths. All values are in $\AA$. Note that no CS and CX errors can be determined with the DFTB2/matsci LC-DFTB2/ob2 approaches is due to the lack of parameters for sulfur and halogens in these parameterizations. The total numbers of reference GS/ES parameters are 31/37 for $\mathrm{CC}, 11 / 11$ for $\mathrm{CO}, 9 / 10$ for $\mathrm{CN}, 25 / 32$ for $\mathrm{CH}, 5 / 5$ for $\mathrm{CS}$, and 8/8 for CX bonds, respectively.

Let us now look at the DFTB statistical results. First, we note that using the matsci parameters instead of their mio counterparts in DFTB2 yields either equal or larger errors, which could be expected as the mio parameters are specifically designed to describe molecules and not materials like the former. As in addition matsci parameters are not available for sulfur and halogen atoms, we focus our discussion on the DFTB2/mio, DFTB3/3ob and LC-DFTB2/ob2 results in the following.

For the GS parameters, Table 3 shows that the deviations are generally larger with DFTB than with both B3LYP and CC2. However, the average errors obtained with the DFTB schemes are rather satisfying. For all tested DFTB approaches, the MAE are indeed ca. $0.01 \AA$ for $\mathrm{CC}, \mathrm{CO}$, and $\mathrm{CS}$ bonds and $1-2^{\circ}$ for valence angles. In contrast, the $\mathrm{CH}$ bonds 
seem problematic for these methods: the computed distances are significantly larger than the reference values, a problem also encountered with the PBE functional. Clearly the LCDFTB2 and DFTB3 approaches provide a significant improvement as compared to DFTB2 (MSE of $+0.024 \AA$ with DFTB2/mio, $+0.013 \AA$ with DFTB3/3ob, and $+0.010 \AA$ with LC-DFTB2/ob2). Whether or not these larger errors for the CH bonds with DFTB than with B3LYP are a significant difficulty or not does, of course, depend on the targeted applications. For the CN bonds, both DFTB2/mio and LC-DFTB2/ob2 provide quite excellent results, actually outperforming $\mathrm{CC} 2$ on the MAE, whereas DFTB3/3ob does not provide an accurate description with rather large overestimations of the bond lengths. For carbon-halogen (CX) distances, the reverse trend is found, with rather limited deviations with DFTB3/3ob and very large errors when using the CX interactions from the halorg set to complete the DFTB2/mio model (MSE of $+0.064 \AA$ ). By considering all bond types, we obtain STD of 0.023, 0.031 and $0.009 \AA$ with DFTB2/mio, DFTB3/3ob, and LC-DFTB2/ob2, respectively, values that are 3-4 times larger than with B3LYP $(0.007 \AA)$ for the two non-LC approaches, indicating a larger spread of the deviations with these schemes. This is graphically illustrated in the left panel of Figure 2 for CO bonds: while B3LYP (CC2) systematically delivers too contracted (extended) carbonyl bonds, the sign of the error might change from one compound to the other with the two DFTB schemes. Note that LC-DFTB2 is more coherent than the other DFTB apporoaches but also yields different error signs for $\mathrm{CO}$ bonds depending on the selected system, e.g., $+0.014 \AA$ for maleimide, but $-0.002 \AA$ for formaldehyde. As a conclusion, using DFTB for determining the GS geometrical parameters of organic compounds is a valid approach, and one can likely advise the use of the LC-DFTB2/ob2 approach, which clearly emerge as competitive for $\mathrm{CNOH}$ compounds, or DFTB3/3ob if halogens are present in the compound. In addition, given its negligible computational cost compared to B3LYP or CC2, DFTB turns out to be "competitive". Indeed, if the spread of the errors is larger with DFTB than with ab initio methods, the DFTB average deviations for the three "molecular" parameterizations remain of the same order of magnitudes as with these ab initio approaches 
for many bond types but for the $\mathrm{CH}$ and CX (with DFTB2/mio) or CN (with DFTB3/3ob) linkages that are less accurately reproduced.
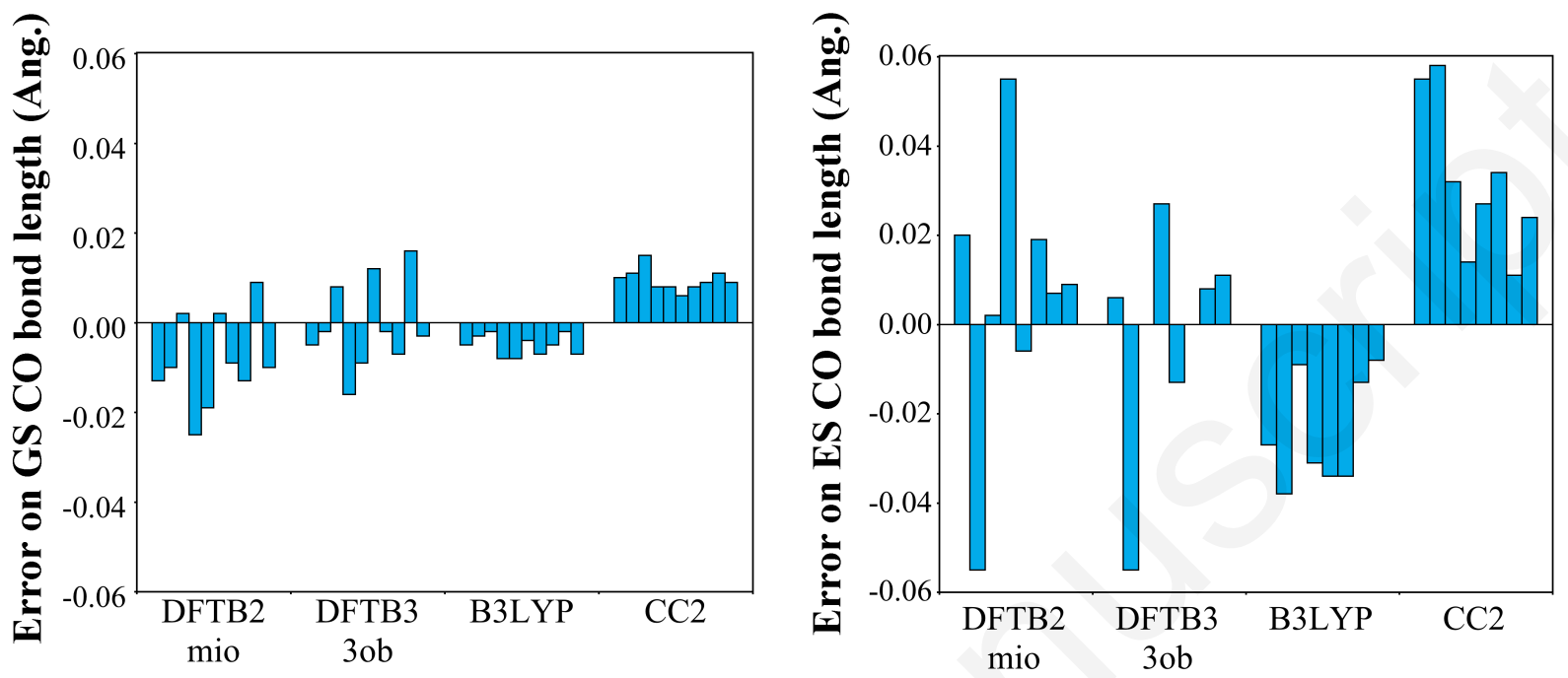

Figure 2: Representation of the errors obtained for the 10 (GS, left) and 8 (ES, right) CO bonds lengths for which all four methods lead to an optimal geometry. Each histogram represents the error made for a specific molecule, ordered in alphabetical order.

As for B3LYP and CC2, the deviations computed for the (LC-)DFTB2 geometrical parameters with respect to reference values are larger in the ES than in GS. Surprisingly, as can be seen in Figure 1, the picture is less clear for DFTB3 with improvement or degradation of the accuracy in the ES as compared to the GS depending on the considered bond type. With all DFTB approaches, the valence angles show typical errors of $2.4-4.1^{\circ}$, whereas the dihedral angles (related to ES puckering here) deviate by $8-10^{\circ}$ on average but with a quite large spread for these two parameters. For both families of angles, the DFTB results are significantly less satisfying than the DFT and CC2 ones that show twice smaller MAE than DFTB. Additionally we notice a totally systematic tendency of underestimating the puckering angles with CC2, i.e., a "consistent" error, whereas as illustrated above for the formaldehyde series, a more inconsistent behavior is found with DFTB. DFTB2/matsci delivers deviations of ca. $0.02-0.03 \AA$ for all bond types for which parameters are available. With DFTB2/mio, the errors on $\mathrm{CC}, \mathrm{CO}, \mathrm{CN}$, and $\mathrm{CH}$ bonds are ca. 0.01-0.02 $\AA$ in the ES, values that can 
probably be viewed as rather satisfying, but quite large errors are found for both the CS (too short, MSE of $-0.040 \AA$ ) and CX (too long, MSE of $+0.059 \AA$ ) bonds. The large errors obtained for CS bonds might be due to the parametrization for the sulfur atoms, as discussed elsewhere. $\stackrel{50}{ }$ Consequently, the STD determined for DFTB2/mio ES bond lengths reaches $0.024 \AA$, significantly above its B3LYP counterpart (0.013 $\AA)$. With both DFTB3/3ob and LC-DFTB2/ob2, the obtained errors in the ES are smaller than the $0.02 \AA$ threshold for all bond types; the two methods even clearly outperforming CC2 for both CO bond lengths in terms of average accuracy. Although this is an additional indication that benchmarking DFTB accuracy on carbonyl bond lengths using B3LYP or CC2 is not a satisfying procedures it should be stressed again that the errors made with DFTB are less systematic than with $a b$ initio methods, as illustrated in the right panel of Figure 2 for CO bonds. In short, for ES optimization, one can most probably select DFTB3/3ob or LC-DFTB2/ob2 as the average errors tend to be smaller than with the two other DFTB parametrizations.

\section{Conclusions and Outlook}

To the best of our knowledge, this work stands as the first in-depth comparison between the GS and ES structural parameters of organic molecules obtained with DFTB and with stateof-the-art wavefunction methods. Such benchmark strategy allows an improved assessment of the DFTB pros and cons, as one is freed from the limitations inherent to the use of reference data coming from experiment (availability, measurement procedures) or TD-DFT (accuracy, choice of a specific functional). Using a batch of 30 molecules exhibiting low-lying $n \rightarrow \pi^{\star}$ and $\pi \rightarrow \pi^{\star}$ electronic transitions, various heteroatoms $(\mathrm{O}, \mathrm{N}$, and $\mathrm{S})$ and halogens $(\mathrm{F}, \mathrm{Cl}$, and $\mathrm{Br}$ ), as well as diverse amplitudes of geometry changes between the GS and ES, the accuracy of four different SCC-DFTB models (DFTB2/mio, DFTB/matsci, DFTB3/3ob and LC-DFTB2/ob2) is assessed and compared to the ones of DFT and CC2.

If the average errors (MAE) are found to be larger for DFTB model than for B3LYP 
and CC2 for the ground state, the picture is less clear for the excited state for which both DFTB3 and LC-DFTB2 sometimes outperforms ab inito methods. As for B3LYP and CC2, the DFTB ability to accurately describe the structures of organic molecules appears to be dependent on the bond type. For the GS, while both CC and CO distances are satisfyingly reproduced, the DFTB geometries show constantly overestimated $\mathrm{CH}$ distances, especially in the DFTB2 framework. In contrast, DFTB2 provides relatively accurate CN distances when DFTB3 returns too long CN bonds. When halogen atoms are present, the DFTB2 distances are largely overestimated and the use of DFTB3 appears necessary. For the GS geometry of compounds with only $\mathrm{C}, \mathrm{N}, \mathrm{O}$ and $\mathrm{H}$ atoms, one can probably suggest the use of the LC-DFTB2 approach which provides the smallest average errors and standard deviations amongst the four tested DFTB models. Interestingly, the accuracy of DFTB does not systematically decrease when going from the GS to the ES description. This could be an advantage as compared to most ab inito methods. However, these errors appear to be less systematic with DFTB than with B3LYP and CC2, showing larger spread, and consequently making trends in a homologous series of chemical systems sometimes inaccurate. In addition, this work also clearly confirms that assessing the accuracy of "low-order" computational schemes without very accurate reference data might be risky, which is in line with a recent work by one of us. $\frac{78}{78}$

As an outlook, we provide in the last Section of the SI a comparison between DFTB3/3ob, LC-DFTB2/ob2, and CC2 ground and excited state structures for five real-life dyes presenting either charge-transfer or highly-delocalized ES. Although CC2 is not accurate enough to provide a quantitative assessment (see above), it is obvious from the data of Table S42 that the two tested DFTB approaches generally reproduce well the evolution of the bond distances induced by electronic excitation. 


\section{Acknowledgement}

The authors are indebted to Prof. Y. Nishimoto for providing the GAMESS code allowing to performed the LC-TD-DFTB2 calculations presented here. D.J. acknowledges the Région des Pays de la Loire for financial support. This research has been performed with resources from i) GENCI-CINES/IDRIS; ii) CCIPL (Centre de Calcul Intensif des Pays de Loire); and iii) Troy cluster installed in Nantes.

\section{Supporting Information Available}

Reference geometries. All considered geometrical parameters.

\section{References}

(1) Adamo, C.; Jacquemin, D. The calculations of Excited-State Properties with TimeDependent Density Functional Theory. Chem. Soc. Rev. 2013, 42, 845-856.

(2) Laurent, A. D.; Adamo, C.; Jacquemin, D. Dye Chemistry with Time-Dependent Density Functional Theory. Phys. Chem. Chem. Phys. 2014, 16, 14334-14356.

(3) Porezag, D.; Frauenheim, T.; Köhler, T.; Seifert, G.; Kaschner, R. Construction of Tight-Binding-Like Potentials on the Basis of Density-Functional Theory: Application to Carbon. Phys. Rev. B 1995, 51, 12947-12957.

(4) Seifert, G.; Porezag, D.; Frauenheim, T. Calculations of Molecules, Clusters, and Solids with a Simplified LCAO-DFT-LDA Scheme. Int. J. Quantum Chem. 1996, 58, 185-192.

(5) Elstner, M.; Porezag, D.; Jungnickel, D.; Elsner, J.; Haugk, M.; Frauenheim, S., Th.and Suhai; Seifert, G. Self-consistent-charge density-functional tight-binding method for simulations of complex materials properties. Phys. Rev. B 1998, 58, 72607268. 
(6) Seifert, G.; Joswig, J.-O. Density-Functional Tight Binding - An Approximate DensityFunctional Theory Method. Wiley Interdiscip. Rev.: Comput. Mol. Sci. 2012, 2, 456-465.

(7) Gaus, M.; Cui, Q.; Elstner, M. DFTB3: Extension of the Self-Consistent-Charge DensityFunctional Tight-Binding Method (SCC-DFTB). J. Chem. Theory Comput. 2011, 7, $931-948$.

(8) Gaus, M.; Goez, A.; Elstner, M. Parametrization and Benchmark of DFTB3 for Organic Molecules. J. Chem. Theory Comput. 2013, 9, 338-354.

(9) Sieck, A.; Frauenheim, T.; Jackson, K. A. Shape Transition of Medium-Sized Neutral Silicon Clusters. Phys. Status Solidi B 2003, 240, 537-548.

(10) Lourenço, M. P.; de Oliveira, C.; Oliveira, A. F.; Guimarãs, L.; Duarte, H. A. Structural, Electronic, and Mechanical Properties of Single-Walled Chrysotile Nanotube Models. J. Phys. Chem. C 2012, 116, 9405-9411.

(11) Krüger, T.; Elstner, M.; Schiffels, P.; Frauenheim, T. Validation of the Density-Functional Based Tight-Binding Approximation Method for the Calculation of Reaction Energies and Other Data. J. Chem. Phys. 2005, 122, 114110.

(12) Gaus, M.; Cui, Q.; Elstner, M. Density Functional Tight Binding: Application to Organic and Biological Molecules. Wiley Interdiscip. Rev.: Comput. Mol. Sci. 2014, 4, $49-61$.

(13) Dolgonos, G.; Aradi, B.; Moreira, N. H.; Frauenheim, T. An Improved Self-ConsistentCharge Density-Functional Tight-Binding (SCC-DFTB) Set of Parameters for Simulation of Bulk and Molecular Systems Involving Titanium. J. Chem. Theory Comput. 2010, 6, $266-278$.

(14) Fihey, A.; Hettich, C.; Touzeau, J.; Maurel, F.; Perrier, A.; Köhler, C.; Aradi, B.; Frauen- 
heim, T. SCC-DFTB Parameters for Simulating Hybrid Gold-Thiolates Compounds. J. Comput. Chem. 2015, 36, 2075-2087.

(15) Niehaus, T.; Elstner, M.; Frauenheim, T.; Suhai, S. Application of an Approximate Density-Functional Method to Sulfur Containing Compounds. J. Mol. Struct.: THEOCHEM 2001, 541, 185-194.

(16) Yang, Y.; Yu, H.; York, D.; Elstner, M.; Cui, Q. Description of Phosphate Hydrolysis Reactions with the Self-Consistent-Charge Density-Functional-Tight-Binding (SCCDFTB) Theory. 1. Parameterization. J. Chem. Theory Comput. 2008, 4, 2067-2084.

(17) Kubar, T.; Bodrog, Z.; Gaus, M.; Köhler, C.; Aradi, B.; Frauenheim, T.; Elstner, M. Parametrization of the SCC-DFTB Method for Halogens. J. Chem. Theory Comput. 2013, 9, 2939-2949.

(18) Lukose, B.; Kuc, A.; Frenzel, J.; Heine, T. On the Reticular Construction Concept of Covalent Organic Frameworks. Beilstein J. Nanotechnol. 2010, 1, 60-70.

(19) Gemming, S.; Enyashin, A. N.; Frenzel, J.; Seifert, G. Adsorption of Nucleotides on the Rutile (110) Surface. Int. J. Mater. Res. 2010, 101, 758-764.

(20) Gaus, M.; Lu, X.; Elstner, M.; Cui, Q. Parameterization of DFTB3/3ob for Sulfur and Phosphorus for Chemical and Biological Applications. J. Chem. Theory Comput. 2014, $10,1518-1537$.

(21) Kubillus, M.; Kubař, T.; Gaus, M.; Řzáč, J.; Elstner, M. Parameterization of the DFTB3 Method for Br, Ca, Cl, F, I, K, and Na in Organic and Biological Systems. J. Chem. Theory Comput. 2015, 11, 332-342.

(22) Lu, X.; Gaus, M.; Elstner, M.; Cui, Q. Parametrization of DFTB3/3ob for Magnesium and Zinc for Chemical and Biological Applications. J. Phys. Chem. B 2015, 119, $1062-1082$. 
(23) Humeniuk, A.; Mitrić, R. Long-Range Correction for Tight-Binding TD-DFT. J. Chem. Phys. 2015, 143, 134120.

(24) Lutsker, V.; Aradi, B.; Niehaus, T. A. Implementation and Benchmark of a LongRange Corrected Functional in the Density Functional Based Tight-Binding Method. J. Chemical Phys. 2015, 143, 184107.

(25) Vuong, V. Q.; Akkarapattiakal Kuriappan, J.; Kubillus, M.; Kranz, J. J.; Mast, T.; Niehaus, T. A.; Irle, S.; Elstner, M. Parametrization and Benchmark of Long-Range Corrected DFTB2 for Organic Molecules. J. Chem. Theory Comput. 2018, 14, 115-125.

(26) Niehaus, T. A.; Suhai, S.; Della Sala, F.; Lugli, P.; Elstner, M.; Seifert, G.; Frauenheim, T. Tight-Binding Approach to Time-Dependent Density-Functional Response Theory. Phys. Rev. B 2001, 63, 085108.

(27) Elstner, M.; Hobza, P.; Frauenheim, T.; Suhai, S.; Kaxiras, E. Hydrogen Bonding and Stacking Interactions of Nucleic Acid Base Pairs: A Density-Functional-Theory Based Treatment. J. Chem. Phys. 2001, 114, 5149-5155.

(28) Petraglia, R.; Steinmann, S. N.; Corminboeuf, C. A Fast Charge-Dependent AtomPairwise Dispersion Correction for DFTB3. Int. J. Quantum Chem. 2015, 115, 12651272.

(29) Barone, V.; Carnimeo, I.; Scalmani, G. Computational Spectroscopy of Large Systems in Solution: The DFTB/PCM and TD-DFTB/PCM Approach. J. Chem. Theory Comput. 2013, 9, 2052-2071.

(30) Nishimoto, Y. Time-Dependent Long-Range-Corrected Density-Functional TightBinding Method Combined with the Polarizable Continuum Model. J. Phys. Chem. A 2019, 123, 5649-565. 
(31) Heringer, D.; Niehaus, T. A.; Wanko, M.; Frauenheim, T. Analytical Excited State Forces for the Time-Dependent Density-Functional Tight-Binding Method. J. Comput. Chem. 2007, 28, 2589-2601.

(32) Fan, G.; Zhu, S.; Xu, H. Density-Functional Theory Study of the Interaction Mechanism and Optical Properties of Flavonols on the Boron Nitride Nanotubes. Int. J. Quantum Chem. 2018, 118, e25514.

(33) Rapacioli, M.; Simon, A.; Marshall, C. C. M.; Cuny, J.; Kokkin, D.; Spiegelman, F.; Joblin, C. Cationic Methylene-Pyrene Isomers and Isomerization Pathways: Finite Temperature Theoretical Studies. J. Phys. Chem. A 2015, 119, 12845-12854.

(34) Nishimoto, Y. DFTB/PCM Applied to Ground and Excited State Potential Energy Surfaces. J. Phys. Chem. A 2016, 120, 771-784, PMID: 26761635.

(35) Domínguez, A.; Aradi, B.; Frauenheim, T.; Lutsker, V.; Niehaus, T. A. Extensions of the Time-Dependent Density Functional Based Tight-Binding Approach. J. Chem. Theory Comput. 2013, 9, 4901-4914.

(36) Nishimoto, Y. Time-Dependent Density-Functional Tight-Binding Method with the Third-Order Expansion of Electron Density. J. Chem. Phys. 2015, 143, 094108.

(37) Kranz, J. J.; Elstner, M.; Aradi, B.; Frauenheim, T.; Lutsker, V.; Garcia, A. D.; Niehaus, T. A. Time-Dependent Extension of the Long-Range Corrected Density Functional Based Tight-Binding Method. J. Chem. Theory Comput. 2017, 13, 1737-1747.

(38) Wanko, M.; Garavelli, M.; Bernardi, F.; Niehaus, T. A.; Frauenheim, T.; Elstner, M. A Global Investigation of Excited State Surfaces Within Time-Dependent DensityFunctional Response Theory. J. Chem. Phys. 2004, 120, 1674-1692.

(39) Job, V.; Sethuraman, V.; Innes, K. The $3500 \AA{ }^{1} \mathrm{~A}_{2}-\mathrm{X}^{1} \mathrm{~A}_{1}$ Transition of Formaldehyde- 
$\mathrm{h}_{2}, \mathrm{~d}_{2}$, and hd: Vibrational and Rotational Analyses. J. Mol. Spectrosc. 1969, 30, $365-426$.

(40) Clouthier, D. J.; Ramsay, D. A. The Spectroscopy of Formaldehyde and Thioformaldehyde. Annu. Rev. Phys. Chem. 1983, 34, 31-58.

(41) Bremond, E.; Savarese, M.; Adamo, C.; Jacquemin, D. Accuracy of TD-DFT Geometries: a Fresh Look. J. Chem. Theory Comput. 2018, 14, 3715-3727.

(42) Christiansen, O.; Koch, H.; Jørgensen, P. Perturbative Triple Excitation Corrections to Coupled Cluster Singles and Doubles Excitation Energies. J. Chem. Phys. 1996, 105, $1451-1459$.

(43) Christiansen, O.; Koch, H.; Jørgensen, P. The Second-Order Approximate Coupled Cluster Singles and Doubles Model CC2. Chem. Phys. Lett. 1995, 243, 409-418.

(44) Christiansen, O.; Koch, H.; Jørgensen, P. Response Functions in the CC3 Iterative Triple Excitation Model. J. Chem. Phys. 1995, 103, 7429-7441.

(45) Andersson, K.; Malmqvist, P. A.; Roos, B. O.; Sadlej, A. J.; Wolinski, K. Second-Order Perturbation Theory With a CASSCF Reference Function. J. Phys. Chem. 1990, 94, $5483-5488$.

(46) Andersson, K.; Malmqvist, P.-A.; Roos, B. O. Second-Order Perturbation Theory With a Complete Active Space Self-Consistent Field Reference Function. J. Chem. Phys. 1992, 96, 1218-1226.

(47) Budzák, Š.; Scalmani, G.; Jacquemin, D. Accurate Excited-State Geometries: a CASPT2 and Coupled-Cluster Reference Database for Small Molecules. J. Chem. Theory Comput. 2017, 13, 6237-6252.

(48) Jacquemin, D. What is the Key for Accurate Absorption and Emission Calculations ? Energy or Geometry? J. Chem. Theory Comput. 2018, 14, 1534-1543. 
(49) Aradi, B.; Hourahine, B.; Frauenheim, T. DFTB+, a Sparse Matrix-Based Implementation of the DFTB Method. J. Phys. Chem. A 2007, 111, 5678-5684.

(50) Petraglia, R.; Corminboeuf, C. A Caveat on SCC-DFTB and Noncovalent Interactions Involving Sulfur Atoms. J. Chem. Theory Comput. 2013, 9, 3020-3025.

(51) Yang,; Yu, H.; York, D.; Cui, Q.; Elstner, M. Extension of the Self-Consistent-Charge Density-Functional Tight-Binding Method: Third-Order Expansion of the Density Functional Theory Total Energy and Introduction of a Modified Effective Coulomb Interaction. J. Phys. Chem. A 2007, 111, 10861-10873.

(52) Perdew, J. P.; Burke, K.; Ernzerhof, M. Generalized Gradient Approximation Made Simple. Phys. Rev. Lett. 1996, 77, 3865-3868.

(53) Becke, A. D. Density-Functional Thermochemistry. 3. The Role of Exact Exchange. J. Chem. Phys. 1993, 98, 5648-5652.

(54) Stephens, P. J.; Devlin, F. J.; Chabalowski, C. F.; Frisch, M. J. Ab Initio Calculation of Vibrational Absorption and Circular Dichroism Spectra Using Density Functional Force Fields. J. Phys. Chem. 1994, 98, 11623-11627.

(55) Barone, V.; Orlandini, L.; Adamo, C. Proton Transfer in Model Hydrogen-Bonded Systems by a Density Functional Approach. Chem. Phys. Lett. 1994, 231, 295-300.

(56) Stephens, P. J.; Devlin, F. J.; Frisch, M. J.; Chabalowski, C. F. Ab initio Calculation of Vibrational Absorption and Circular Dichroism Spectra Using Density Functional Force Fields. J. Phys. Chem. 1994, 98, 11623-11627.

(57) Hättig, C.; Weigend, F. CC2 Excitation Energy Calculations on Large Molecules Using the Resolution of the Identity Approximation. J. Chem. Phys. 2000, 113, 5154-5161.

(58) Hättig, C.; Hald, K. Implementation of RI-CC2 Triplet Excitation Energies With an Application to Trans-Azobenzene. Phys. Chem. Chem. Phys. 2002, 4, 2111-2118. 
(59) Frisch, M. J.; Trucks, G. W.; Schlegel, H. B.; Scuseria, G. E.; Robb, M. A.; Cheeseman, J. R.; Scalmani, G.; Barone, V.; Petersson, G. A.; Nakatsuji, H.; Li, X.; Caricato, M.; Marenich, A. V.; Bloino, J.; Janesko, B. G.; Gomperts, R.; Mennucci, B.; Hratchian, H. P.; Ortiz, J. V.; Izmaylov, A. F.; Sonnenberg, J. L.; Williams-Young, D.; Ding, F.; Lipparini, F.; Egidi, F.; Goings, J.; Peng, B.; Petrone, A.; Henderson, T.; Ranasinghe, D.; Zakrzewski, V. G.; Gao, J.; Rega, N.; Zheng, G.; Liang, W.; Hada, M.; Ehara, M.; Toyota, K.; Fukuda, R.; Hasegawa, J.; Ishida, M.; Nakajima, T.; Honda, Y.; Kitao, O.; Nakai, H.; Vreven, T.; Throssell, K.; Montgomery, J. A., Jr.; Peralta, J. E.; Ogliaro, F.; Bearpark, M. J.; Heyd, J. J.; Brothers, E. N.; Kudin, K. N.; Staroverov, V. N.; Keith, T. A.; Kobayashi, R.; Normand, J.; Raghavachari, K.; Rendell, A. P.; Burant, J. C.; Iyengar, S. S.; Tomasi, J.; Cossi, M.; Millam, J. M.; Klene, M.; Adamo, C.; Cammi, R.; Ochterski, J. W.; Martin, R. L.; Morokuma, K.; Farkas, O.; Foresman, J. B.; Fox, D. J. Gaussian 16 Revision A.03. 2016; Gaussian Inc. Wallingford CT.

(60) TURBOMOLE V6.6 2014, a development of University of Karlsruhe and Forschungszentrum Karlsruhe GmbH, 1989-2007, TURBOMOLE GmbH, since 2007; available from http://www.turbomole.com (accessed 13 June 2016).

(61) Moule, D. C.; Foo, P. D. Analysis of the $2973 \AA$ Absorption System of Phosgene. J. Chem. Phys. 1971, 55, 1262-1268.

(62) Judge, R.; Moule, D. The $\tilde{A}^{1} A^{\prime \prime} \leftarrow \tilde{X}^{1} A^{\prime}$ Electronic Transition in Formyl Chloride, CHClO. J. Mol. Spectrosc. 1985, 113, 302-309.

(63) Ding, H.; J. Orr-Ewing, A.; N. Dixon, R. Rotational Structure in the $\tilde{A}^{1} A^{\prime \prime}-\tilde{X}^{1} A^{\prime}$ Spectrum of Formyl Chloride. Phys. Chem. Chem. Phys. 1999, 1, 4181-4185.

(64) Ernsting, N. P.; Pfab, J.; Romelt, J. Geometry Changes Accompanying Electronic Excitation of Nitrosomethane in the $650 \mathrm{~nm}$ Region. J. Chem. Soc., Faraday Trans. 2 1978, 74, 2286-2294. 
(65) Gordon, R. D.; Luck, P. Conformational Changes Accompanying Electronic Excitation of $\mathrm{CD}_{3}$ NO. Chem. Phys. Lett. 1979, 65, 480-483.

(66) Judge, R.; Moule, D. Thiocarbonyl Spectroscopy: The $\tilde{A}^{1} A^{\prime \prime} \leftarrow \tilde{X}^{1} A^{\prime}$ and $\tilde{a}^{3} A^{\prime \prime} \leftarrow \tilde{X}^{1} A^{\prime}$ Electronic Transitions in Thioformyl Chloride, CHCIS. J. Mol. Struct. 1985, 113, 77-84.

(67) Bokarev, S. I.; Dolgov, E. K.; Bataev, V. A.; Godunov, I. A. Molecular Parameters of Tetraatomic Carbonyls $\mathrm{X}_{2} \mathrm{CO}$ and $\mathrm{XYCO}(\mathrm{X}, \mathrm{Y}=\mathrm{H}, \mathrm{F}, \mathrm{Cl})$ in the Ground and Lowest Excited Electronic States, Part 1: A Test of Ab Initio Methods. Int. J. Quantum Chem. 2009, 109, 569-585.

(68) Jensen, P.; Bunker, P. The Geometry and the Inversion Potential Function of Formaldehyde in the $\tilde{\mathrm{A}}^{1} \mathrm{~A}_{2}$ and $\tilde{\mathrm{a}}^{3} \mathrm{~A}_{2}$ Electronic States. J. Mol. Spectrosc. 1982, 94, 114-125.

(69) Dallos, M.; Müller, T.; Lischka, H.; Shepard, R. Geometry Optimization of Excited Valence States of Formaldehyde Using Analytical Multireference Configuration Interaction Singles and Doubles and Multireference Averaged Quadratic Coupled-Cluster Gradients, and the Conical Intersection Formed by the $1{ }^{1} B_{1}\left(\sigma-\pi^{*}\right)$ and $2^{1} A_{1}\left(\pi-\pi^{*}\right)$ States. $J$. Chem. Phys. 2001, 114, 746-757.

(70) Hubbard, L. M.; Bocian, D. F.; Birge, R. R. The Nature of the ${ }^{1} n \pi^{\star} \leftarrow S_{0}$ Transition. 4. The First Excited Singlet State of Acetaldehyde. J. Am. Chem. Soc. 1981, 103, $3313-3320$.

(71) Guido, C. A.; Jacquemin, D.; Adamo, C.; Mennucci, B. On the TD-DFT Accuracy in Determining Single and Double Bonds in Excited-State Structures of Organic Molecules. J. Phys. Chem. A 2010, 114, 13402-13410.

(72) Bousquet, D.; Fukuda, R.; Maitarad, P.; Jacquemin, D.; Ciofini, I.; Adamo, C.; Ehara, M. Excited-State Geometries of Heteroaromatic Compounds: A Comparative TD-DFT and SAC-CI Study. J. Chem. Theory Comput. 2013, 9, 2368-2379. 
(73) Guareschi, R.; Filippi, C. Ground- and Excited-State Geometry Optimization of Small Organic Molecules with Quantum Monte Carlo. J. Chem. Theory Comput. 2013, 9, $5513-5525$.

(74) Brémond, É.; Savarese, M.; Su, N. Q.; Pérez-Jiménez, Á. J.; Xu, X.; Sancho-García, J. C.; Adamo, C. Benchmarking Density Functionals on Structural Parameters of Small/Medium-Sized Organic Molecules. J. Chem. Theory Comput. 2016, 12, 459-465.

(75) Tuna, D.; Lu, Y.; Koslowski, A.; Thiel, W. Semiempirical Quantum-Chemical Orthogonalization-Corrected Methods: Benchmarks of Electronically Excited States. J. Chem. Theory Comput. 2016, 12, 4400-4422.

(76) Köhn, A.; Hättig, C. Analytic Gradients for Excited States in the Coupled-Cluster Model CC2 Employing the Resolution-Of-The-Identity Approximation. J. Chem. Phys. 2003, 119, 5021-5036.

(77) Hättig, C. In Response Theory and Molecular Properties (A Tribute to Jan Linderberg and Poul Jørgensen); Jensen, H. A., Ed.; Advances in Quantum Chemistry; Academic Press, 2005; Vol. 50; pp 37-60.

(78) Suellen, C.; Garcia Freitas, R.; Loos, P.-F.; Jacquemin, D. Cross Comparisons Between Experiment, TD-DFT, CC, and ADC for Transition Energies. J. Chem. Theory Comput. 2019, doi: 10.1021/acs.jctc.9b00446. 


\section{Graphical TOC Entry}

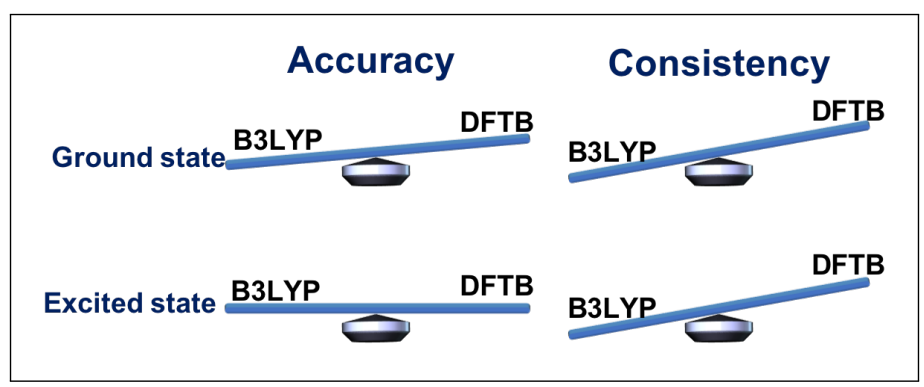

55

56

57

58 